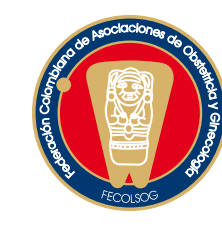

\title{
Editorial
}

\section{EL PAPEL DE LAS SOCIEDADES CIENTÍFICAS Y LA ACADEMIA EN LA ACTUALIZACIÓN PERMANENTE DEL PLAN DE BENEFICIOS EN EL SISTEMA DE SEGURIDAD SOCIAL EN SALUD EN COLOMBIA}

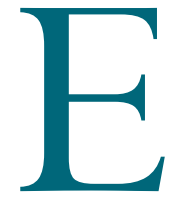

l plan de beneficios ha sido definido como un "conjunto de actividades, procedimientos, suministros y reconocimientos que se brinda a las personas, con el propósito de mantener o recuperar la salud y evitar el menoscabo de su capacidad económica derivada de la incapacidad temporal por enfermedad general, maternidad y la incapacidad, discapacidad o invalidez derivada de los riesgos de accidentes de trabajo y enfermedad profesional". ${ }^{1}$

En Colombia la ley 100 creó inicialmente tres planes de beneficios: el plan de atención básica $(\mathrm{PAB})$, el plan obligatorio de salud contributivo (POS) y el plan obligatorio de salud subsidiado (POSS), diferentes en su origen y en los servicios que proporcionan a la población beneficiaria. La sentencia T 760 de 2008 de la Corte Constitucional ordenó, entre otros aspectos, los relacionados con el plan de beneficios: "(i) adoptar medidas para eliminar la incertidumbre acerca del contenido de los planes de beneficios y lograr la actualización periódica de los mismos; (ii) unificar los planes de beneficios (POS y POSS), primero en el caso de los niños y, luego, progresivamente en el caso de los adultos, teniendo en cuenta su adecuada financiación”. ${ }^{2}$

De acuerdo con esta sentencia, el antiguo Ministerio de la Protección Social, hoy en día Ministerio de Salud y Protección Social, comenzó a dar una serie de pasos para cumplir lo ordenado por la Corte Constitucional. En ese sentido, el Ministerio divulga en el año 2009, a petición de la Comisión de Regulación en Salud (CRES), el documento para la actualización integral de los planes obligatorios de salud. ${ }^{3}$ En este documento se plantean como elementos centrales en la actualización del plan de beneficios los siguientes aspectos: la priorización de las problemáticas en salud que deben ser abordadas por el plan de beneficios, el desarrollo de las guías de atención integral basadas en la evidencia y la evaluación de tecnologías en salud.

Con el término "tecnologías de la salud" se denomina cualquier intervención que pudiera ser utilizada para: promover la salud, prevenir, diagnosticar o tratar la enfermedad o para la rehabilitación en el cuidado a largo plazo. Incluye los medicamentos, dispositivos médicos, procedimientos y sistemas organizacionales utilizados en el cuidado de la salud. ${ }^{4}$ Estos no son excluyentes entre sí y en la práctica diaria pueden usarse simultáneamente: un procedimiento quirúrgico como el reemplazo total de cadera puede involucrar el uso de dispositivos médicos (prótesis) y medicamentos (anestésicos, antibióticos). Lo anterior pone en evidencia que el plan de beneficios está estrechamente ligado a las tecnologías de la salud.

Las tecnologías en la salud han determinado, en gran medida, el incremento en la expectativa de vida de las personas que se ha observado desde los años setenta ${ }^{5}$ y el incremento en los costos de la atención médica en los últimos años en todos los países. Por ejemplo, en el año 2000 el gasto en salud en relación con el PIB en los Estados Unidos fue del 13,5 \%, mientras que en el año 2006 fue del 15,3\%. Para este 
período en Colombia representó el 7,8\%, en Chile, el 5,3\%, en México, el 6,6\% y en Perú, el 4,4\%. ${ }^{6}$

En Colombia, el incremento en los costos de las tecnologías que se utilizan en el tratamiento de algunas patologías, muchas de ellas no incluidas en el plan de beneficios, por lo que originaban recobros que debían ser cubiertos por el Fondo de Solidaridad y Garantía (FOSYGA), fue el argumento que se consideró inicialmente responsable de la crisis financiera que afectó al sector de la salud en el año 2009 y que motivó la expedición del decreto 4975 de 2009, por el cual se proclamó la emergencia social, ${ }^{7}$ decreto que posteriormente fue declarado inexequible por la Corte Constitucional por la sentencia C 252 de $2010 .{ }^{8}$

Vale la pena recordar que los verdaderos responsables de la crisis del sector de la salud han sido, en primer lugar, la corrupción, en segundo lugar, la falta de vigilancia por parte de los entes de control y, en tercer lugar, la falta de voluntad política de los organismos reguladores, ${ }^{9}$ lo cual condujo a que se cediera ante las presiones de los grupos de interés, que obraron en beneficio propio y, de esa manera, se produjo la crisis de recursos en el sector. Estos grupos corresponden a: aquellos que son responsables de la producción de estas tecnologías, quienes las dispensan, quienes las prescriben y, por último, quienes son sus beneficiarios, los pacientes. ${ }^{10}$

Para afrontar la crisis de una manera efectiva se requiere que la sociedad como un todo esté vigilante y ejerza control sobre todos los actores del sistema, como lo plantea de manera interesante el doctor Javier Eslava en su artículo sobre la corrupción en el sector salud. Además se requiere un cambio en la manera como la sociedad colombiana valora las vías para obtener dinero. Se requiere la reconstrucción del concepto de lo que es un bien público, entendido como "un bien que está disponible a todos y del cual el uso por una persona no substrae de su uso por otros". Este esfuerzo deberá partir, como lo puntualiza Eslava, desde la familia y el sector educativo.

Por otra parte, se requiere fortalecer las entidades que regulan y controlan el sector para que ejerzan control sobre los precios a las tecnologías disponibles y a los márgenes de ganancia de las empresas que participan del mercado de la salud. ${ }^{10}$ También se necesita el uso de metodologías sistemáticas para la evaluación de las tecnologías en salud. Este aspecto es tomado en cuenta por la ley 1438 de 2011, que en su artículo 92 plantea la creación del Instituto de Evaluación Tecnológica en Salud. ${ }^{12}$

La evaluación de tecnologías sanitarias es definida como "la sistemática evaluación de las propiedades, efectos y otros impactos de las intervenciones en el cuidado de la salud. Su principal propósito es informar el proceso de toma de decisiones en la atención en salud ya sea a nivel individual, de la institución, del cobrador o a nivel local, regional o internacional". ${ }^{13}$ Es un campo multidisciplinario que aborda el impacto de las tecnologías en la salud, considera el contexto específico en que se brinda la atención en salud, como también las alternativas disponibles. ${ }^{14}$ Implica que las observaciones para un escenario de atención en salud dirigidas a un individuo, una población o país no son necesariamente válidos en otros contextos.

La evaluación de tecnologías juzga e informa sobre los aspectos relacionados con el uso de la tecnología, tales como la eficacia, efectividad, seguridad, propiedades técnicas, impacto económico, ético, social y legal, y sus implicaciones políticas. Por tener un papel clave en la toma informada de decisiones, se fundamenta en la aplicación de la medicina basada en la evidencia, entendida como el uso de la mejor evidencia disponible a partir de la investigación científica y médica, acompañada de la aplicación de la experiencia clínica y la observación en la atención individual del paciente. Utiliza una amplia variedad de métodos, entre ellos la investigación primaria, ya sea experimental, a partir de ensayos clínicos aleatorizados, observacionales, secundarios o integrativos, como las revisiones sistemáticas, los metaanálisis y las evaluaciones económicas disponibles; o a partir de diferentes fuentes de información presentes en diferentes escenarios. ${ }^{13}$

Uno de los puntos más importantes en la evaluación de tecnologías es la transparencia con la que se realiza en aspectos tales como la búsqueda e 
identificación no sesgada de la evidencia relevante, la priorización de los temas a ser evaluados y la declaración de los potenciales conflictos de interés que puedan resultar en sesgos en la evaluación. 13,15

La evaluación de tecnologías presenta desafíos importantes cuando se hace en escenarios de recursos restringidos, entre ellos, la falta de investigadores que estén adecuadamente capacitados en el uso de las metodologías que la soportan, la limitación de recursos económicos, por lo que es preferible edificar economías a escala que permitan reducir costos y, por último, lograr que los procesos tengan la transparencia que asegure su credibilidad y validez a todos los interesados y no respondan de manera sesgada a los intereses de algunos grupos.

Para responder a estos desafíos, la Universidad de Antioquia, la Universidad Javeriana y la Universidad Nacional de Colombia han conformado la alianza Centro de Investigación y Evaluación de Tecnologías en Salud (CINETS). Estas instituciones congregan un importante número de investigadores con experiencia en las metodologías que fundamentan la evaluación de tecnologías, documentada por múltiples publicaciones en este campo. Desarrollan actualmente, en conjunto con varias sociedades científicas, entre ellas Fecolsog, nueve de las trece guías de atención integral ${ }^{16}$ y participan en redes internacionales en el tema como la Health Technology Assesment International (HTAi), la Colaboración Cochrane y la Guidelines Internacional Network (GIN), el National Institute for Clinical Excelence (NICE), solo por mencionar algunas.

Esta alianza busca apoyar al Instituto de Evaluación de Tecnologías del gobierno nacional, a los gobiernos locales y a las instituciones que lo requieran, en la toma de decisiones informadas respecto a las tecnologías en salud que pretendan implementar, en la elaboración de reportes en salud pública (policy briefs), como también en el desarrollo de guías de práctica clínica. Busca dar la mayor calidad, independencia y eficiencia a las evaluaciones requeridas. También busca realizar actividades de capacitación a otras instituciones universitarias, instituciones hospitalarias y a las sociedades científicas.
La participación de las sociedades científicas, de la Academia Nacional de Medicina y de la Federación Médica Colombiana, no se limita a recibir capacitación, sino que también incluye la participación activa en los organismos a los cuales han sido designados, como el mencionado Instituto de Evaluación Tecnológica; la participación activa en la generación de las mejores propuestas y la constitución de los equipos de trabajo para responder a la urgente necesidad del país en la construcción de las guías y los reportes de evaluación de tecnologías y, por último, en la vigilancia y control social, para que la Comisión de Regulación en Salud, el Ministerio de Salud y Colciencias hagan convocatorias transparentes, de acuerdo con las necesidades del país. En nuestro caso es necesario que se priorice el campo de la salud sexual, reproductiva, materna y perinatal.

Corresponde al Ministerio de Salud, a la CRES y a Colciencias liderar de manera diáfana estos nuevos procesos que marcarán la ruta para dar el mejor plan de beneficios a la población colombiana con criterios de calidad, eficiencia y equidad. Corresponde a las sociedades científicas y la academia construir las propuestas que aseguren que la inversión vaya en beneficio de la población colombiana y no termine en manos de los corruptos, como ya ha pasado.

Por último, en aras de la transparencia, hago mi declaración de los posibles conflictos de interés en el tema, como miembro de la Federación Colombiana de Obstetricia y Ginecología y profesor de la Universidad Nacional de Colombia.

\section{Hernando Gaitán}

Editor

Profesor Titular

Departamento de Obstetricia y Ginecología

Universidad Nacional de Colombia

\section{REFERENCIAS}

1. Universidad de Antioquia. Aprende en línea. Curso de Seguridad Social en Salud. Definición de plan de beneficios. Visitado 2011 Dic 29. Disponible en: http://aprendeenlinea.udea.edu.co/lms/moodle/ course/view.php?id=319 
2. Corte Constitucional. Sentencia T 760 de 2008. Visitado 2011 Dic 29. Disponible en: http://www. corteconstitucional.gov.co/relatoria/2008/T-760-08.htm

3. Ministerio de la Protección Social. Documento para la garantía y protección del derecho a la salud de los colombianos. Actualización integral de los Planes Obligatorios de Salud. 2009. Visitado 2011 Dic 29. Disponible en: http://www.pos.gov.co/Paginas/ Documentost\%C3\%A9cnicos-POS.aspx

4. INATHA. HTA Resources. Visitado 2011 Dic 29. Disponible en: http://www.inahta.org/HTA/

5. World Bank. Life Expectancy. Visitado 2011 Dic 23. Disponible en: http://www.worldbank.org/depweb/ english/modules/social/life/index.html

6. Organización Mundial de la Salud. Estadísticas Sanitarias Mundiales 2009. ISBN 978924356381 Visitado 2011 Dic 29. Disponible en: http://www.who. int/whosis/whostat/ES_WHS09_Full.pdf

7. Presidencia de la República de Colombia. Ministerio de la protección social. Decreto 4975 del 23 de Diciembre de 2009

8. Corte Constitucional. Sentencia C 262 del 16 de Abril de 2010. Visitado 2011 Dic 23. Disponible en: http:// www.actualicese.com/normatividad/2009/12/23/ decreto-4975-de-23-12-2009/

9. Revista Semana. Recobros en salud, un desfalco anunciado. Edición del 3 de Mayo de2011. Visitado 2011 Dic 29. Disponible en: http:// www.semana.com/nacion/recobros-salud-desfalcoanunciado/156077-3.aspx

10. Ess SM, Schneeweiss S, Szucs TD. European healthcare policies for controlling drug expenditure. Pharmacoeconomics 2003;21:89-103

11. Ostrom E. Glosario sobre el Conocimiento como bien común. Visitado 2011 Dic 29. Disponible en: http:// gruizlegal.blogspot.com/2009/10/elinor-ostromnobel-de-economia.html

12. Congreso de Colombia. Ley 1438 de 2011. Diario Oficial No. 47.957 de 19 de enero de 2011. Visitado 2011 Dic 29. Disponible en: http://www. secretariasenado.gov.co/senado/basedoc/ley/2011/ ley_1438_2011.html

13. Resources for HTA - HTAi and INAHTA's White Paper to WHO. Visitado 2011 Dic 29. Disponible en: http://www.inahta.org/HTA/

14. INATHA. Health Technology Assessment (HTA) Glossary. Visitado 2011 Dic 29. Disponible en: http:// www.inahta.org/GO-DIRECT-TO/Members/

15. Lavis JN, Permanand G, Oxman AD, Lewin S, Fretheim A. SUPPORT Tools for evidence-informed health Policymaking (STP) 13: Preparing and using policy briefs to support evidence-informed policymaking. Health Res Policy Syst 2009;7:S13.

16. Centro de Investigación y Evaluación de Tecnologías en Salud - CINETS. Guías de Atención Integral. Disponible en: http://www.alianzacinets.org/ 